\title{
Variación espacial y temporal en el oleaje del Mar de Bellingshausen
}

\author{
Spatial and temporal variations in \\ waves of Bellingshausen Sea
}

Manuel Contreras ${ }^{1}$, Matías Alday ${ }^{2}$,

Jimmy Ceballos ${ }^{3} \&$ Tito Erazo ${ }^{3}$

\section{Resumen}

El propósito de este trabajo fue presentar una caracterización de las variaciones espaciales y temporales del oleaje en aguas profundas en el Mar de Bellingshausen. Para cumplir este objetivo se usaron cuatro series de oleaje reconstruido a partir del modelo global Wave Watch III, calibrado con altimetría satelital. Las series tienen una longitud de 14 años con los parámetros de resumen de estados de mar cada 3 horas. Se constata que la altura significativa del oleaje en el Mar de Bellingshausen disminuye cuando la latitud aumenta desde los $60^{\circ} \mathrm{S}$ a los $66^{\circ} \mathrm{S}$. Simultáneamente, la dirección peak pasa de ser predominantemente WSW - W a WNW - W, en el mismo sentido de variación de la latitud. Por último, la altura significativa aumenta linealmente, mientras que la frecuencia de ocurrencia de los eventos extremos en el sector norte de la zona de estudio, aumentó en los últimos años del período analizado.

\section{Palabras Clave:}

Cambio climático contemporáneo, altura significativa y dirección peak del oleaje, reconstrucción de oleaje.

\section{Abstract}

The purpose of this paper was to present a characterization of the spatial and temporal variations of deep water waves in the Bellingshausen Sea. To meet this objective, four series of reconstructed waves were used from the Wave Watch III global model calibrated with satellite altimetry. The series have a length of 14 year with the statistical parameters of sea states every 3 hours. It is observed that the

significant wave height in the Bellingshausen Sea decreases as latitude increases from $60^{\circ} \mathrm{S}$ to $66^{\circ}$ S. Simultaneously, the peak direction changes from predominantly WSW - W to $\mathrm{W}-\mathrm{W}$, in the same sense of variation of latitude. Finally, the significant height increases linearly, while the frequency of occurrence of extreme events in the northern part of the study area increased in the last years of the analyzed period.

\section{Key Words:}

Contemporary climate change, significant wave height, peak wave direction, Hindcasting.

\section{INTRODUCCIÓN}

El oleaje es uno de los fenómenos más característicos del comportamiento de la superficie de los océanos. Este se encuentra determinado principalmente por la acción del viento sobre la superficie del mar como acción generadora y la gravedad como fuerza restauradora (Massel, 1996). Se caracteriza por los parámetros: 1) altura $(\mathrm{H})$, que corresponde a la distancia vertical entre la cresta y el

1 Facultad de Ingeniería, Investigador Centro de Estudios Avanzados, Universidad de Playa Ancha.

manuel.contreras@upla.cl $\bowtie$

2 Escuela de Ingeniería Oceánica, Facultad de Ingeniería, Universidad de Valparaíso. matias.alday@gmail.com

3 Facultad de Ciencias Agropecuarias y Departamento de Medio Ambiente, Universidad Laica "Eloy Alfaro" de Manabí. jimmycev@hotmail.com 
valle de la onda; 2) periodo (T), que señala el tiempo que transcurre entre el paso de dos crestas o valles consecutivos por un mismo punto; y 3) dirección (Dir), que indica la orientación con la que incide la trayectoria de la onda.

Debido al cambio climático contemporáneo, se ha pronosticado que el oleaje experimentará un aumento en su altura (Mori et al. 2010; Izaguirre et al. 2011; Wang et al. 2014) y cambios en la dirección (Hemer, 2010; Hemer et al. 2010; Molina, 2011; Molina et al. 2011; Molina \& Contreras, 2012). Recientes trabajos buscan estimar la magnitud de estos cambios en diferentes océanos (Stopa \& Cheung, 2014) y los posibles efectos asociados en diferentes costas del mundo (Sierra \& Casas-Prat, 2014). Sin embargo no existen estudios sobre el comportamiento de las variaciones del oleaje en el Mar de Bellingshausen.

Como las aguas del Océano Austral rodean todo el globo, influyen en las dinámicas atmosféricas y oceanográficas que regulan el clima del planeta (Lumpkin \& Speer, 2007). Por otra parte, la Península Antártica es una de las regiones más afectadas por el cambio climático (Turner et al. 2009; Turner et al. 2014), al experimentar un aumento en las temperaturas atmosféricas hasta seis veces superiores al promedio mundial observado en las últimas décadas (Contreras-López \& Cevallos, 2014). Surge así la pregunta si el cambio y la variabilidad climática que afecta el planeta, se encuentra alterando también el clima del oleaje en el Mar de Billingshausen.

De esta forma, el propósito de este trabajo es presentar una caracterización de las variaciones espaciales y temporales del oleaje en aguas profundas que se encuentra frente a la Península Antártica, en las inmediaciones de la base científica Pedro Vicente Maldonado (62 $\left.27^{\circ} \mathrm{S} ; 5^{\circ} 44,5^{\prime} \mathrm{W}\right)$, en isla Greenwich, de manera de estimar una primera aproximación sobre los efectos del calentamiento del planeta sobre el oleaje en esta zona.

\section{MATERIALES Y MÉTODOS}

Para analizar los cambios espaciales $y$ temporales del oleaje en aguas profundas de la Península Antártica, se usaron cuatro series de tiempo de estados de mar estimados cada 3 horas, a partir del modelo global Wave Watch III (Komen et al. 1994;
Tolman, 2009), que consisten en reconstrucciones históricas a partir de campos de viento, modelos atmosféricos y validados con altimetría satelital, con una resolución de $1^{\circ}$ en latitud y $1,25^{\circ}$ en longitud para todo el planeta. Las series utilizadas poseen una longitud de 14 años, comprendiendo la ventana temporal entre el 30 de enero de 1997 y el 1 de enero de 2011. El modelo global Wave Watch III ha sido comparado con otros modelos de reconstrucción del oleaje en el océano, mostrando un mejor rendimiento en la reconstrucción del oleaje (Hanson et al. 2009). El campos de viento y los datos de validación son proporcionados por el National Centers for Environmental Prediction-National Center for Atmospheric Research, NCEP-NCAR (Kalnay et al. 1996).

Cada serie de tiempo corresponde a los estados de mar reconstruidos en los nodos cuya posición geográfica se detalla en la tabla 1 . Un estado de mar se define como aquella situación o periodo de tiempo en que, y debido a la inercia del fenómeno, se considera que existe un cierto equilibrio entre las fuerzas generadoras y las fuerzas restauradoras que intervienen, lo cual produce que su manifestación permanezca aproximadamente en estado estacionario (Ochi, 1998). Así, en las variaciones de periodo corto del oleaje, del orden de segundos, el proceso se considera estacionario y corresponde a lo que se encuentra en un estado de mar; mientras que las variaciones de periodo largo, del orden de horas, se evoluciona de un estado de mar a otro. Esto permite caracterizar cada estado de mar con estadígrafos de resumen. En este trabajo se considera para caracterizar cada estado de mar a:

- La altura significativa (Hsig).

- El período peak.

- La dirección peak.

En la figura 1 se muestra el emplazamiento relativo de los nodos con respecto a la estación científica Pedro Vicente Maldonado.

Se usaron los algoritmos getgrib.m y read_grib. $\mathrm{m}^{1}$ implementados para Matlab versión R2013a para extraer los parámetros de resumen en los 4 nodos seleccionados.

\footnotetext{
1 Disponible en <http://polar.ncep.noaa.gov/waves/ensemble/ download.shtml?text> [fecha visita: diciembre de 2014].
} 


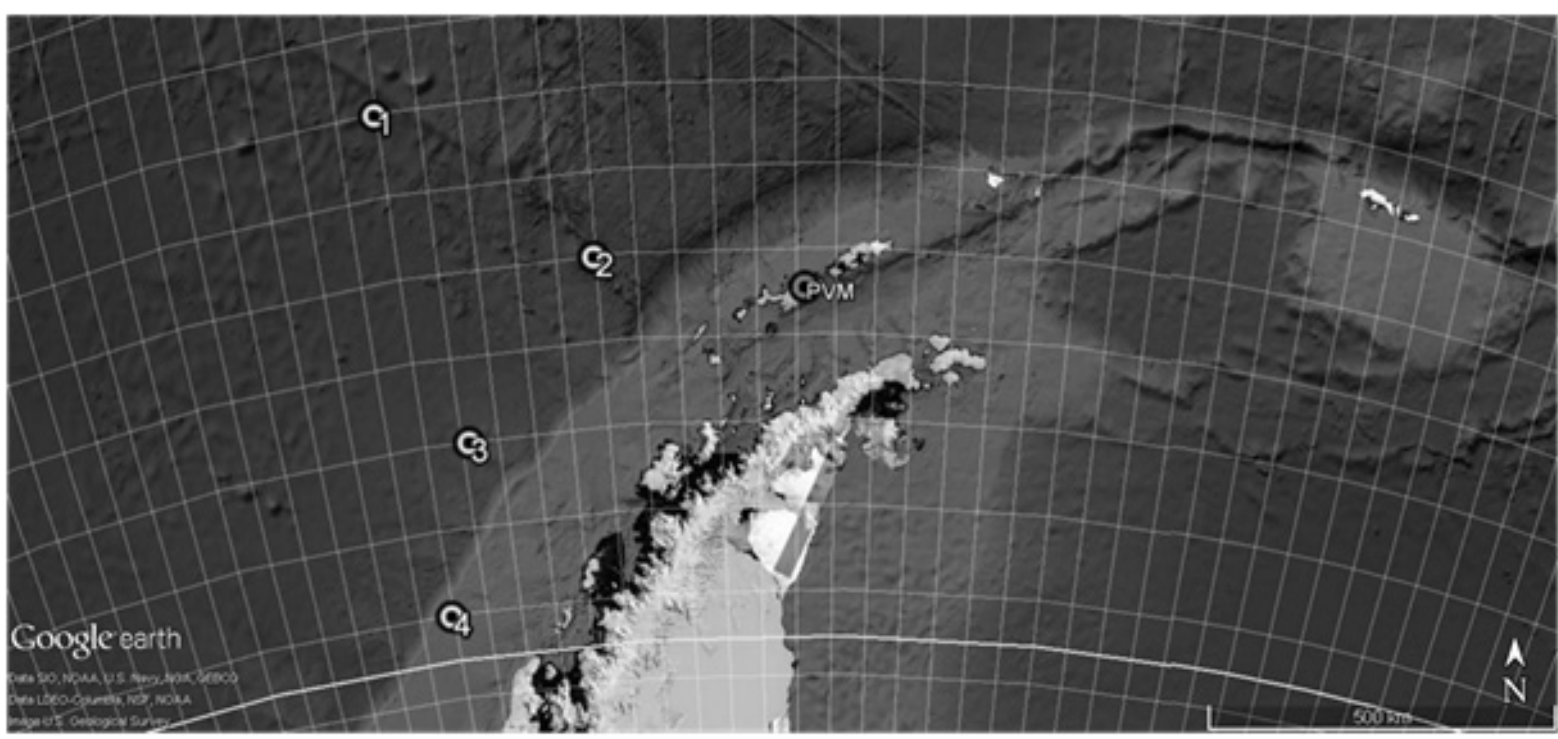

Fig. 1. Distribución nodos para el análisis del oleaje en aguas profundas del Mar de Bellingshauser en las cercanías de la estación científica Pedro Vicente Maldonado, isla Greenwich, Península Antártica.

Se confeccionaron histogramas para la distribución de frecuencias de la altura significativa, dirección peak, y diagramas de dispersión de altura significativa versus dirección.

Las series de tiempo de las alturas significativas para cada uno de los cuatro nodos fue graficada en su evolución temporal, ajustando una tendencia lineal por el método de mínimos cuadrados.

\section{RESULTADOS}

En la figura 2 se muestran los histogramas de distribución de frecuencia de la altura significativa en rangos de $0,5 \mathrm{~m}$. Se puede observar que la moda cambia desde el intervalo 3 - 3,5 $\mathrm{m}$ en la latitud de $60^{\circ} \mathrm{S}$ a $2-2,5 \mathrm{~m}$ en la latitud de $66^{\circ} \mathrm{S}$. Apreciándose también un aumento en las alturas significativas inferiores a $1 \mathrm{~m}$ desde norte a sur y una disminución del oleaje extremo (mayor a 5 metros), también desde norte a sur.

En la figura 3 se muestran los histogramas de distribución de frecuencia de la dirección peak en el Mar de Bellingshauser. La moda en todos los nodos es la dirección $\mathrm{W}$, pero se puede apreciar un cambio en el sesgo de la distribución. La participación de las direcciones S, SSW, SW, WSW disminuyen paulatinamente mientras las componentes WNW, NW y NNW aumentan desde la latitud $60^{\circ} \mathrm{S}$ a la $66^{\circ} \mathrm{S}$. Este cambio en la frecuencia de la dirección del oleaje también es apreciado en los gráficos de la dispersión de la altura significativa versus dirección peak (Fig. 4). En esta figura también se puede apreciar como las alturas significativas extremas (mayor a $5 \mathrm{~m}$ ) se ordenan desde los $60^{\circ} \mathrm{S}$ a los $66^{\circ} \mathrm{S}$, pasando de

Tabla 1. Localización de los nodos usados para analizar el oleaje reconstruido en la península Antártica.

\begin{tabular}{cccc}
\hline $\mathrm{N}^{\circ}$ Nodo & Latitud & Longitud & Descripción \\
\hline 1 & $60^{\circ} \mathrm{S}$ & $70^{\circ} \mathrm{W}$ & Límite Mar de Bellingshausen \\
2 & $62^{\circ} \mathrm{S}$ & $65^{\circ} \mathrm{W}$ & Frente I. Greendwich \\
3 & $64^{\circ} \mathrm{S}$ & $68,75^{\circ} \mathrm{W}$ & Frente I. Anversr \\
4 & $66^{\circ} \mathrm{S}$ & $70^{\circ} \mathrm{W}$ & Frente I. Alejandro I, Círculo Polar Antártico
\end{tabular}




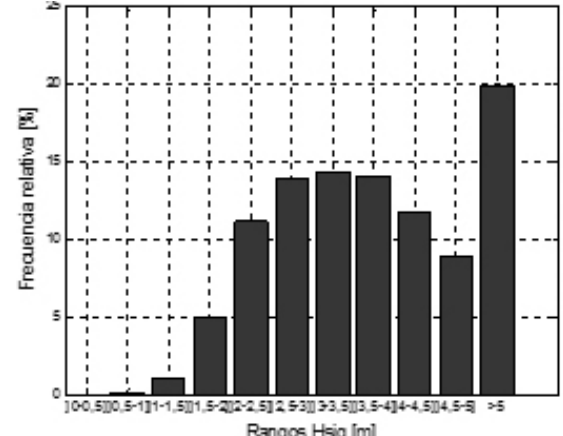

Rangos Hsig [m]

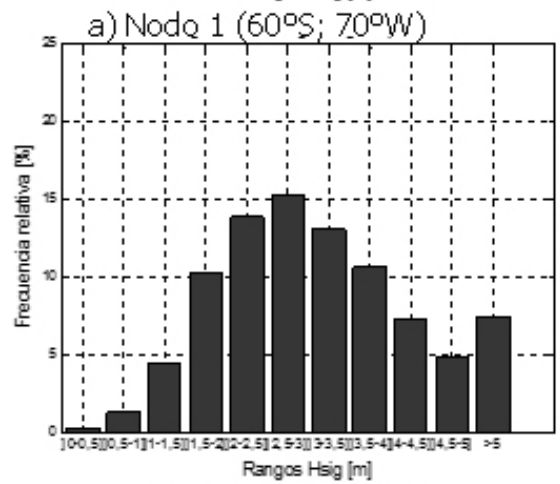

c) Nodo 3 (64ㅇ; $\left.68,75^{\circ} \mathrm{W}\right)$

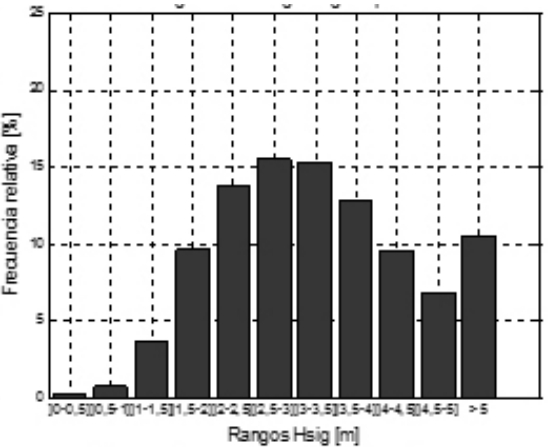

b) Nodo $2(62 \circ 5 ; 65 \circ)$

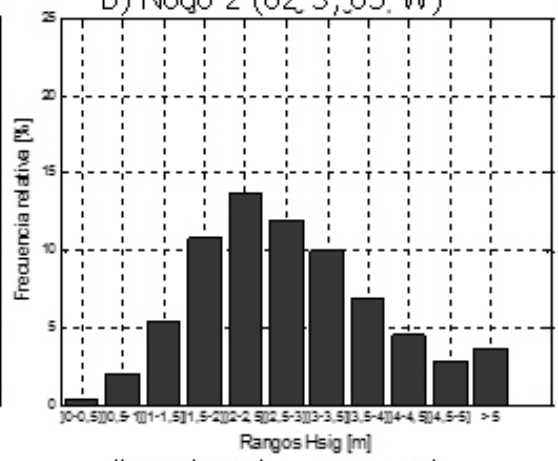

d) Nodo 4 (66 $5 ; 70^{\circ} \mathrm{W}$ )

Fig. 2. Histogramas de distribución de frecuencias de la altura significativa (Hsig) del oleaje en el mar de Bellingshauser.

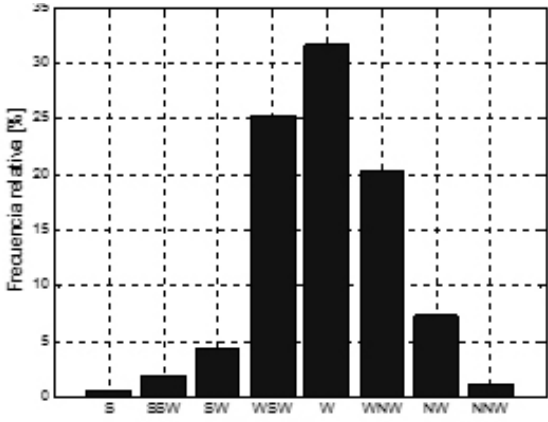

Diección de Incidencia ["]

a) Nodo $1\left(60^{\circ} \mathrm{S} ; 70^{\circ} \mathrm{W}\right)$

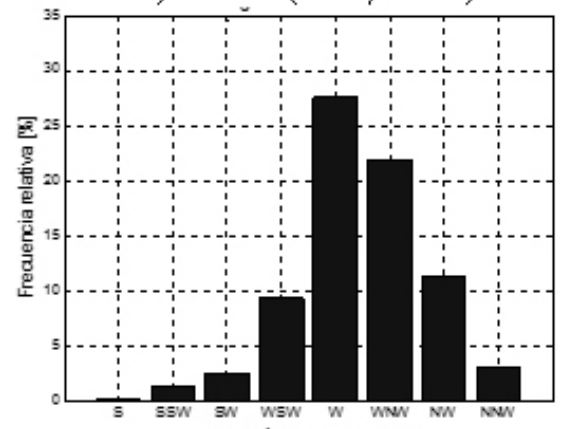

c) Nodo $3\left(64 \circ 5 ; 68,75^{\circ} \mathrm{W}\right)$

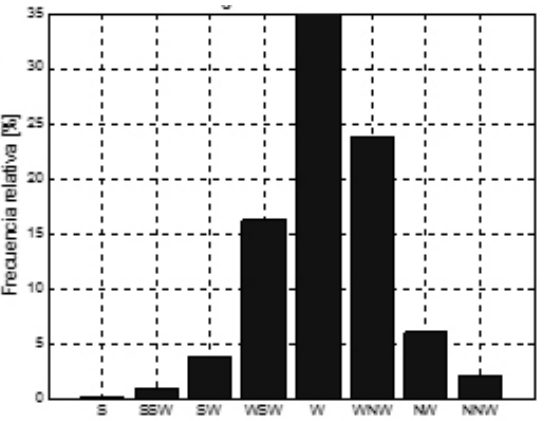

Direcocón de incidencia ["]

b) Nodo $2\left(62^{\circ} \mathrm{S} ; 65^{\circ} \mathrm{W}\right)$

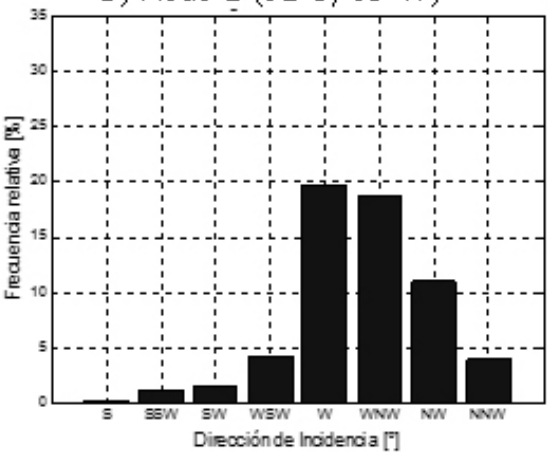

d) Nodo $4\left(66^{\circ} \mathrm{s} ; 70^{\circ} \mathrm{W}\right)$

Fig. 3. Histogramas de distribución de frecuencias de la dirección del oleaje en el mar de Bellingshauser. 


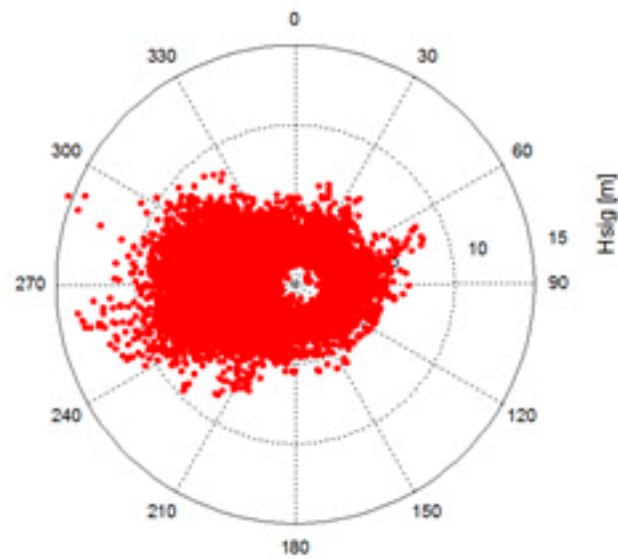

a) Nodo $1\left(60^{\circ} \mathrm{S} ; 70^{\circ} \mathrm{W}\right)$

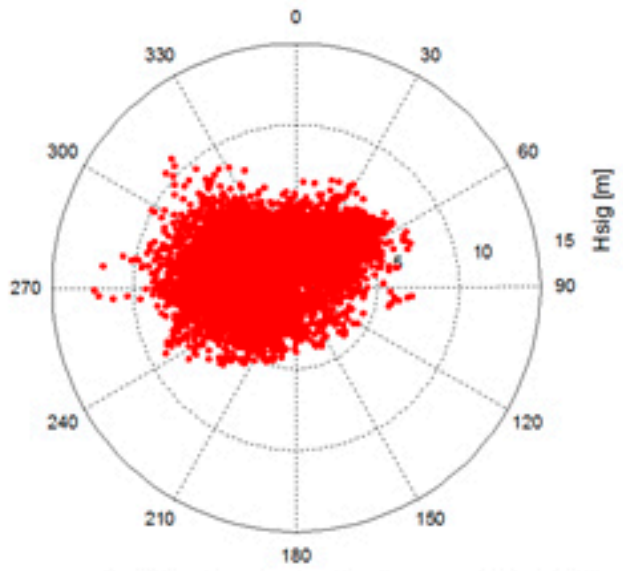

c) Nodo $3\left(64 \circ\right.$; $\left.68,75^{\circ} \mathrm{W}\right)$

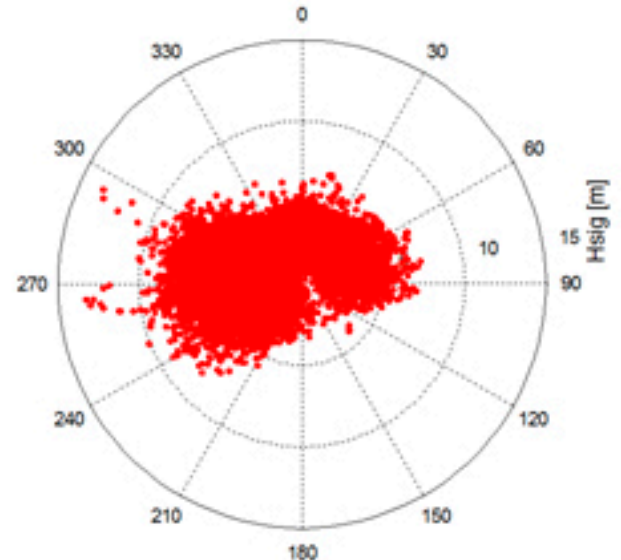

b) Nodo $2\left(62^{\circ}\right.$; $\left.65^{\circ} \mathrm{W}\right)$

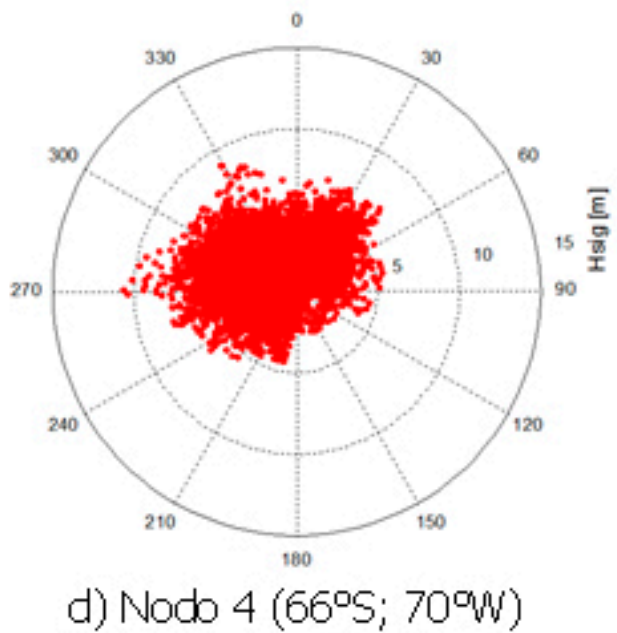

Fig. 4. Dispersión de la altura significativa del oleaje con respecto la dirección del oleaje en el mar de Bellingshauser.

una distribución aleatoria uniforme a una dirección preponderantemente $\mathrm{S}$.

Por último, al observar las series de tiempo de las alturas significativas entre el año 1997 a 2011, desde los $60^{\circ} \mathrm{S}$ a $66^{\circ} \mathrm{S}$, disminuye la ocurrencia de eventos extremos marcada por alturas significativas mayores a $8 \mathrm{~m}$ (Fig. 5). Sin embargo en todos los nodos se puede verificar una tendencia lineal positiva en la altura significativa, al mismo tiempo que la frecuencia de eventos extremos se encuentra aumentando año a año en el período analizado.

\section{DISCUSIÓN}

Los cambios experimentados en el continente Antártico en los últimos años, han motivado una serie de estudios, entre los que destaca el reporte "Antarctic Climate Change and the Environment" (Turner et al. 2014), desarrollado por el comité científico en investigación antártica, donde se analiza el rol del continente antártico en el clima global, la instrumentación y reportes sobre registros y tendencias de diversos parámetros como nivel del mar, biología marina y terrestre, parámetros meteorológicos, observaciones de hielos marinos, capa de hielo y permafrost, entre otros. Sin embargo, aunque en el informe son mencionados o discutidos los efectos e importancia del oleaje marino, este proceso no es considerado como un parámetro para ser analizado en profundidad. Esta omisión resulta peculiar, toda vez que el oleaje es el proceso que explica la mayor cantidad de energía 
a) Nodo $1\left(60^{\circ} \mathrm{S} ; 70^{\circ} \mathrm{W}\right)$

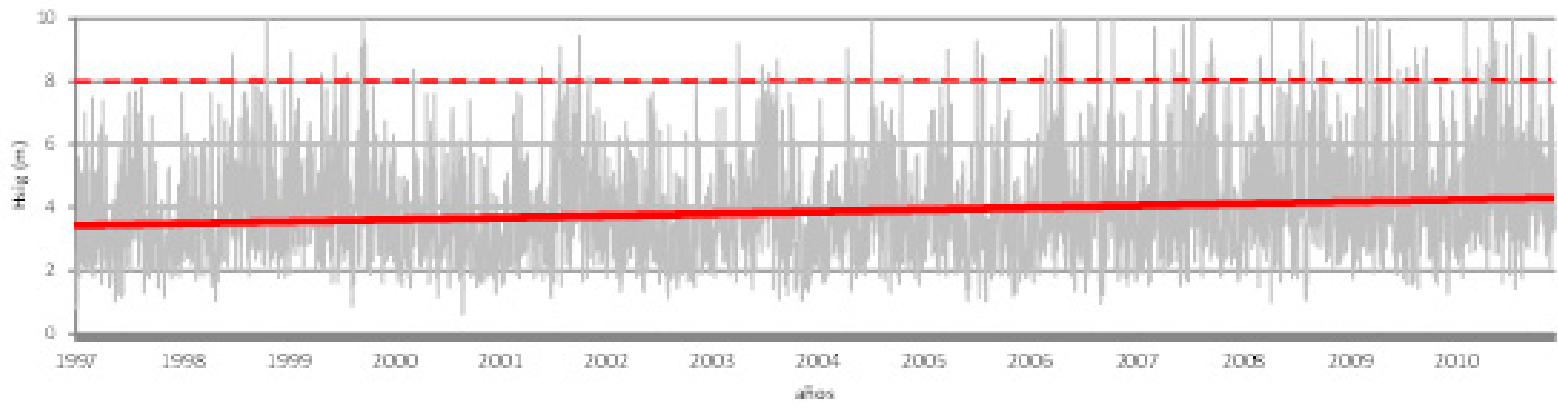

b) Nodo $2\left(62^{\circ} \mathrm{S} ; 65^{\circ} \mathrm{W}\right)$

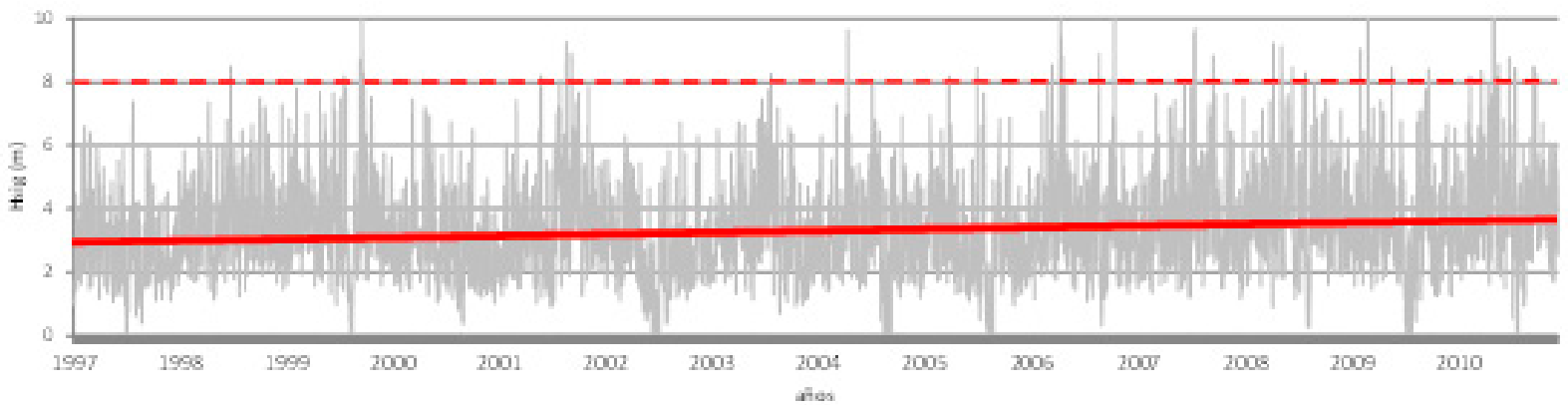

c) Nodo $3\left(64^{\circ} \mathrm{S} ; 68,75^{\circ} \mathrm{W}\right)$

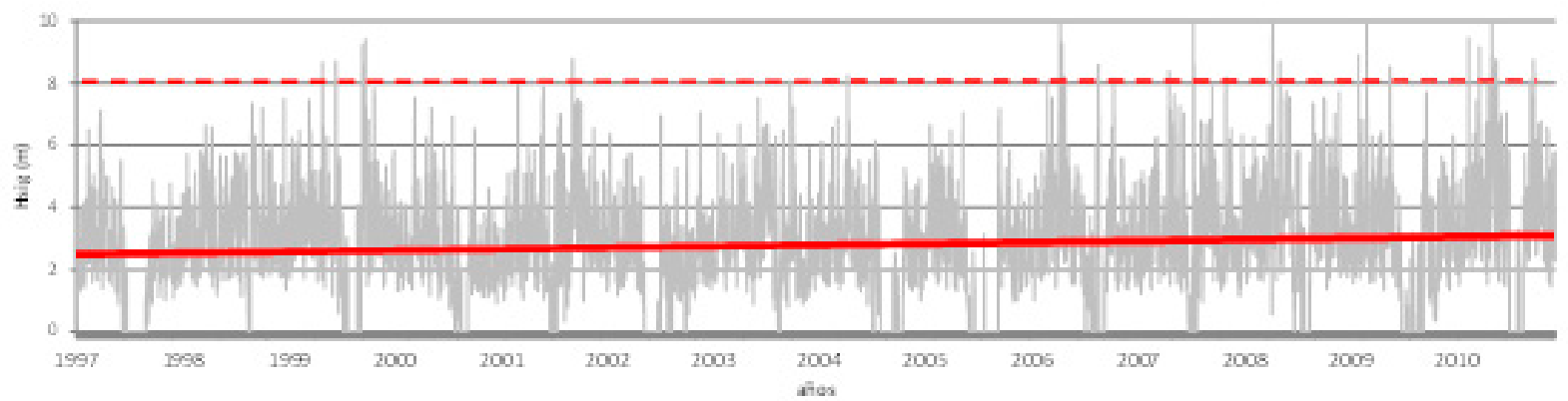

d) Nodo $4\left(66^{\circ} \mathrm{S} ; 70^{\circ} \mathrm{W}\right)$

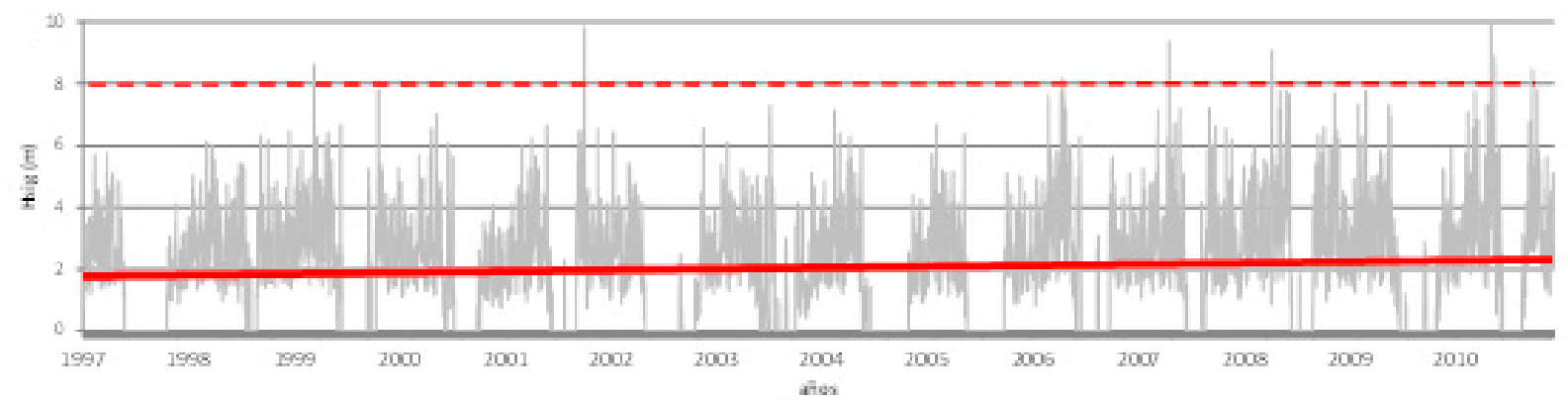

Fig. 5. Series de tiempo de la altura significativa (Hsig) del oleaje entre 1997 y 2011 en el Mar de Bellingshauser. Se muestran las tendencias lineales (línea gruesa continua) ajustadas por el método de mínimos cuadrados. La línea segmentada señala los eventos extremos que superan los $8 \mathrm{~m}$ de altura significativa. 
potencial y variabilidad de la superficie del océano (Massel, 1996; Ochi, 1998). De esta forma, parece natural establecer que la variabilidad climática y el cambio climático se expresen también en alteraciones sobre la propagación del oleaje. Esto fue verificado en las costas de Chile (Molina, 2011; Molina et al. 2011), donde se establece que uno de los efectos del cambio climático sería la alteración de la dirección de incidencia del oleaje (Molina \& Contreras, 2012). Esto significa en la práctica que en el futuro cercano se espera un cambio en los delicados equilibrios hidrodinámicos que sustentan las formaciones de playas, zonas de erosión costera, lugares de convergencia o dispersión de sedimentos, tasas se sedimentación, entre otros, a lo largo de la costa del litoral del Pacífico Sur Oriental (litoral de Colombia, Ecuador, Perú y Chile). Resultados similares han sido obtenidos también en Australia (Hemer, 2010; Hemer et al. 2010).

La verificación de la existencia de cambios espaciales y temporales del oleaje en el Mar de Bellingshausen constituye el punto de partida para relacionar - a través de la propagación del oleaje cómo las alteraciones del calentamiento global en la Antártica pueden estar afectando la propagación y el arribo de las olas de mar de fondo en lugares tan distantes como la costa ecuatoriana (ContrerasLópez \& Cevallos, 2014).

Las variaciones espaciales en el comportamiento del oleaje del Mar de Bellingshauser se pueden atribuir al proceso de generación del oleaje de mar de fondo (fetch del swell del sur).

Debido a que se encuentran variaciones temporales similares a las encontradas en Australia (Hemer et al. 2010) y Chile central (Molina et al. 2011) con series más largas, la variación temporal en el comportamiento del oleaje, sugiere que el cambio climático contemporáneo se encuentra alterando el clima de oleaje en el Mar de Bellingshauser. Sin embargo, se debe recordar que sólo se analizan 14 años de estados de mar, por lo que resulta necesario contar con series más extensas para separar el efecto del cambio climático de variaciones interdecadales, por ejemplo. Una mejora sustantiva para comprender las variaciones que experimenta el oleaje en la Antártica se conseguiría con la instalación de un instrumento que registre en tiempo real el oleaje en estas latitudes.

\section{CONCLUSIONES}

Se constata que los parámetros de resumen de estados del mar de Bellingshausen, consistentes en la altura significativa, período peak y dirección peak, experimentan variaciones espaciales en 6 grados de latitud como temporales en una ventana de 14 años.

Desde los $60^{\circ} \mathrm{S}$ hasta los $66^{\circ} \mathrm{S}$, la altura significativa y la ocurrencia de valores extremos disminuyen, mientras que la dirección peak pasa de ser predominantemente WSW -W a WNW - W.

En 14 años recientes, la altura significativa se aumenta linealmente, mientras que la frecuencia de ocurrencia de los eventos extremos en el sector norte del Mar de Bellingshausen se incrementa.

\section{AGRADECIMIENTOS}

Al Instituto Antártico Ecuatoriano (INAE) por facilitar la participación de JC en las expediciones. A la Secretaría Nacional de Educación Superior, Ciencia, Tecnología e Innovación (SENESCYT) por el financiamiento y apoyo que está dando al proyecto (INAE-I-05-12-2), "Relación de Procesos Físicos del Calentamiento Global y Cambio Climático entre la Antártida y Ecuador".

\section{LITERATURA CITADA}

Contreras-López, M. \& Cevallos, J. (2014). Cambio climático contemporáneo y una conjetura sobre la relación Antártica - Sudamérica a través del oleaje. Hemispheric \& Polar Studies Journal, 5(1), 26-40.

Hanson, J. L., Tracy, B. A., Tolman, H. L. \& Scott, R. D. (2009). Pacific Hindcast Performance of Three Numerical Wave Models. Journal Atmospheric Oceanic Technology, 26, 1614-1633.

Hemer, M., Church, J. \& Hunter, J. (2010). Variability and trends in the directional wave climate of the Southern Hemisphere. International Journal of Climatology, 30(4), 475-491.

Hemer, M. (2010). Historical trends in Southern 
Ocean storminess: Long-term variability of extreme wave heights at Cape Sorell, Tasmania. Geophysical Research Letters, 37, L18601, 5.

Izaguirre, C., Méndez, F.J., Menéndez, M. \& Losada, I. J. (2011). Global extreme wave height variability based on satellite data. Geophysical Research Letters, 38, L10607, 6.

Kalnay, E., Kanamitsu, M., Kistler, R., Collins, W., Deaven, D., Gandin, L., Iredell, M.,... Joseph, D. (1996). The NCEP/NCAR 40-Year Reanalysis Project. Bull. Amer. Meteor. Soc., 77, 437-471.

Komen, J., Cavaleri, L., Donelan, M., Hasselmann, K., Hasselmann, S. \& Janssen, P.A. (1994). Dynamics and Modelling of Ocean Waves. Cambridge University Press.

Lumpkin, R. \& Speer, K. (2007). Global Ocean Meridional Overturning. Journal of Physical Oceanography, 37(10), 2550-2562.

Massel, S.R. (1996). Ocean Surface Waves: Their Physics and prediction. Advanced Series on Ocean Engineering, World Scientific.

Molina, M. (2011). Estudio no estacionario de clima medio de oleaje en la costa central de Chile. Tesis de Ingeniero Civil Oceánico. Valparaíso, Chile: Universidad de Valparaíso.

Molina, M., Contreras, M., Winckler, P., Salinas, S. \& Reyes, M. (2011). Consideraciones sobre las Variaciones de Mediano y Largo Plazo del Oleaje en el Diseño de Obras Marítimas en Chile Central. Anales del Instituto de Ingenieros de Chile, 23(3), 77-88.

Molina, M. \& Contreras, M. (2012). Variabilidad climática y oleaje en el Pacífico Sur Oriental: costa de Chile. Científica, 9(3), 240-250.

Mori, N., Yasuda, T., Mase, H., Tom, T. \& Oku,
Y. (2010). Projection of Extreme Wave Climate Change under Global Warming. Hydrological Research Letter, 4, 15-19.

Ochi, M.K. (1998). Ocean Waves: The Stochastic Approach. Cambridge Ocean Technology Series $\mathrm{N}^{\circ} 6$.

Sierra, J. P. \& Casas-Prat, M. (2014). Analysis of potential impacts on coastal areas due to changes in wave conditions. Climatic Change, 124, 861-876.

Stopa, J. E. \& Cheung, K. F. (2014). Periodicity and patterns of ocean wind and wave climate, Journal of Geophysical Research: Oceans, 119, 5563-5584.

Tolman, H.L. (2009). User manual and system documentation of WAVEWATCH III. TM version 3.14. Technical Note. Environmental Modeling Center Marine Modeling and Analysis Branch. National Oceanic and Atmospheric Administration, NOAA. 194pp. Disponible en línea (fecha última visita en agosto de 2014), <http,//nopp. ncep.noaa.gov/mmab/papers/tn276/ MMAB_276.pdf>.

Turner, J., Bindschadler, R., Convey, P., di Prisco, G., Fahrbach, E., Gutt, J., Hodgson, D., Mayewski, P. \& Summerhayes, C. (2009). Antarctic Climate Change and the Environment. Scientific Committee on Antarctic Research.

Turner, J., Barrand, N.E., Bracegirdle, T.J., Convey, P., Hodgson, D.A., Jarvis, M., Jenkins, A.,... Klepikov, A. (2014). Antarctic climate change and the environment: an update. Polar Record, 50(3), 237-259.

Wang, X.L., Feng, Y. \& Swail, V.R. (2014). Changes in global ocean wave heights as projected using multimodel CMIP5 simulations. Geophysical Research Letters, 41, 1026-1034. 